\title{
CO oxidation on ceria studied by electrochemical impedance spectroscopy
}

\author{
Xavier Vendrel[ ${ }^{[a],[b],}$, , Yuri Kubyshin ${ }^{[a],[c]}$, Lourdes Mestres ${ }^{[d]}$, Jordi Llorca ${ }^{[a],[b]}$ \\ [a] Dr X Vendrell, Dr Y Kubyshin, Prof J Llorca \\ Institute of Energy Technologies \\ Universitat Politècnica de Catalunya, EEBE \\ Eduard Maristany, 10-14, 08019, Barcelona, Spain \\ E-mail: xavier.vendrell.villafruela@upc.edu \\ [b] Dr X Vendrell, Prof J Llorca \\ Department of Chemical Engineering and Barcelona Research Centre in Multiscale Science and Engineering \\ Universitat Politècnica de Catalunya, EEBE \\ Eduard Maristany, 10-14, 08019, Barcelona, Spain \\ [c] Dr Y Kubyshin \\ Department of Physics \\ Universitat Politècnica de Catalunya, EEBE \\ Eduard Maristany, 10-14, 08019, Barcelona, Spain \\ [d] Prof L Mestres \\ Department of Inorganic and Organic Chemistry \\ Universitat de Barcelona \\ Martí i Franquès, 1-11, 08028, Barcelona, Spain
}

Supporting information for this article is given via a link at the end of the document

\begin{abstract}
Electrochemical impedance spectroscopy technique has been widely used to analyze the electrical properties of a large number of materials. In this study, the electrical properties of $\mathrm{CeO}_{2}$ pellets under $\mathrm{CO}$ oxidation conditions have been analyzed by electrochemical impedance spectroscopy. $\mathrm{CeO}_{2}$ pellets have been prepared by a conventional precipitation method and sintered at low temperature to satisfy a compromise between large surface area and a high relative density of the pellet. The electrical properties of $\mathrm{CeO}_{2}$ have been investigated under different atmosphere conditions such as $\mathrm{N}_{2}, \mathrm{O}_{2}, \mathrm{CO}, \mathrm{CO}_{2}$, or selected combinations. The electrical sensitivity of $\mathrm{CeO}_{2}$ to the surrounding atmosphere allows to follow the catalytic reaction as a function of the $\mathrm{CO}: \mathrm{O}_{2}$ ratio and temperature. The appropriate analysis of the electrical response by electrochemical impedance spectroscopy could open a new insight to monitor the catalytic response and behavior of any catalyst.
\end{abstract}

\section{Introduction}

$\mathrm{CeO}_{2}$ or ceria is a well know material exhibiting oxide-ion and electron conduction over a wide range of temperatures ${ }^{[1-4]}$. Therefore, ceria and ceria-based materials are a very versatile family of oxides which have a wide range of applications in a large variety of fields, such in sensors ${ }^{[5-7]}$, solid-oxide fuel cells $(\mathrm{SOFC})^{[8-10]}$, lithium-ion batteries ${ }^{[11-13]}$, photoelectrochemistry and solar cells $s^{[14,15]}$, catalysis ${ }^{[16,17]}$ and other energy-related applications ${ }^{[18,19]}$. The widespread applications of ceria-based materials are determined by the characteristic electronic configuration of cerium and the high stability of its two valence states, $\mathrm{Ce}^{4+}$ and $\mathrm{Ce}^{3+}$. The transformation between the two oxidation states determines the capacity of cerium oxide to release or adsorb oxygen. According to the Kröger-Vink notation the loss of oxygen and the reduction of $\mathrm{Ce}^{4+}$ to $\mathrm{Ce}^{3+}$ is accompanied by the formation of oxygen vacancies, equation 1 : $2 \mathrm{Ce} e_{C e}+\mathrm{O}_{O} \rightarrow V_{o}+2 C e_{C e}^{\prime}+1 / 2 \mathrm{O}_{2}$

The role of oxygen vacancies in the fluorite structure of $\mathrm{CeO}_{2}$ is one of the most important characteristics to achieve high catalytic performance in oxidation reactions. In this sense, in order to indepth investigate the capacity of ceria to gain and lose oxygen, one of the main catalytic reactions that has been widely investigated is the oxidation of $\mathrm{CO}$. Extensive studies have been dedicated to determine the effects of the structure ${ }^{[20,21]}$, morphology ${ }^{[22-24]}$, dopants ${ }^{[25]}$ or supported metals ${ }^{[26-28]}$ on the catalytic oxidation of $\mathrm{CO}$ by ceria. Over the last decade, great interest in understanding the outstanding properties of ceria and ceria-based materials has resulted in a development and use of a large diversity of characterization techniques. The most common being transmission electron microscopy (TEM) to determine the morphology, size and facets exposed; X-ray diffraction and Raman spectroscopy to analyze the structure and determine the presence of oxygen vacancies; nitrogen adsorption/desorption to determine the surface area and pore size distributions, temperature-programmed reduction/oxidation to study the redox characteristics and determine oxygen storage capacity (OSC) and X-ray photoelectron spectroscopy (XPS) to study the surface chemical characteristics, among others. Depending on the application of ceria, more specific techniques such as electron paramagnetic resonance (EPR), cyclic voltammetry (CV), ultraviolet photoelectron spectroscopy (UPS) or electrochemical impedance spectroscopy (EIS) can also be used. EIS is particularly useful to study the electrical and functional properties of different materials ${ }^{[29-31]}$. EIS is a powerful technique that can provide further information on the redox processes occurring at the solid-gas interface surface ${ }^{[32,33]}$. Therefore, the use of EIS in heterogeneous catalysis could elucidate which redox processes govern the chemical reactions and give a definite answer to some of the unknown processes occurring during the solid-gas interactions.

However, the combination of EIS or the analysis of the evolution of the resistance and catalytic tests is rarely seen and only a few examples can be found in the literature ${ }^{[34-38]}$. This is closely related to the fact that different conditions are required for measurements of the electrical properties and the catalytic activity. On one side, for heterogeneous catalytic measurements, samples are usually in the form of powder in order to promote a larger surface contact between the sample and the surrounding gases. On the other side, in order to measure accurately the electrical properties with EIS, samples are commonly in the form of dense ceramics ( $>80 \%$ relative density) to avoid any distortion on the 
measurement. Therefore, measuring the catalytic performance and the electrical properties of a material at the same time presents a great challenge and some considerations must be taken. However, the electrical properties studied in operando conditions on catalysts, so far, are mainly focused on the analysis of the electrical resistance of the catalyst and only at one frequency, moreover, the catalyst was in the form of powder ${ }^{[35]}$. The use of only one frequency to measure the resistance of the catalyst can lead to a misinterpretation of the result. As it is extendedly explained in the supplementary information file and in [39], the use of EIS implies measuring the sample at a wide range of frequencies at different temperatures in order to separate the influence of the different regions that contribute to the overall resistance of the sample. The different regions whose characteristic relaxation time constant, $\mathrm{T}$, is given by the product of $\mathrm{R}$ (resistance) and $\mathrm{C}$ (capacitance) satisfying equations 2 and 3.

$\tau=R C$

$\omega_{\max } R C=1$

In the frequency regime, $\mathrm{RC}$ elements are separable due to the relaxation shown in equation 3 which holds at the frequency maximum loss, $\omega_{\max }$, in the impedance spectrum, where $\omega_{\max }=$ $2 \pi f_{\max }$ and $f_{\max }$ is the frequency corresponding to the maximum of the an impedance arc (see the supplementary information file). Consequently, when determining the resistance of the sample at one single frequency at different temperatures the obtained resistance values give no information of the region that is contributing to the resistance. Furthermore, up to date, the acquisition of the catalyst resistance has been made in the form of powder ${ }^{[35,36]}$, which may lead to errors in the measurement due to possible movement or reorganization of the powder when heating or due to the gas flow. Again, no information concerning different regions that contribute to the global resistance can be extracted. Hence, the use of relatively dense samples is required to extract further and trustable data on electrical properties from the EIS data.

The objectives of the present work are to (1) obtain impedance data on pure $\mathrm{CeO}_{2}$ under different atmosphere and catalytic conditions, (2) find the most appropriate equivalent circuit to model the impedance data, (3) extract values of the various equivalent circuits parameters as a function of temperature and catalytic conditions and (4) monitor the catalytic response by EIS.

\section{Results and Discussion}

Impedance data for $\mathrm{CeO}_{2}$ measured in dry $\mathrm{N}_{2}$ at $450^{\circ} \mathrm{C}$ are shown in Figure 1 as (a) a $Z$ complex plane plot and (b) $C^{\prime}$, (c) $Y^{\prime}$ and (d) $Z$ ''M" spectroscopic plots. At this temperature the impedance data are dominated by the sample response while the sampleelectrode contact impedances start to appear at lower frequencies or at higher temperatures. The complex plane plot (a) shows a semicircle at high frequency (inset) and a partial semicircle at lower frequencies which can be attributed to bulk and grain boundary, respectively. The resistivity values of the bulk $R_{b}$, can be obtained from the intercept of the arc on $Z^{\prime}$ axis, however, the resistivity values of the grain boundary, $R_{g b}$, can only be obtained at higher temperatures. It is worth mentioning that a direct extraction of the resistance from the intercept on $Z$ ' axis may lead to large errors, especially at high temperatures. The different regions that contribute to the total resistance of the sample may not correspond to a perfect semicircle, the relaxation time constants of the different regions may overlap or simply may not be evident ${ }^{[39]}$. $Z^{*}$ plots on linear scales give undue weighting to the largest resistances in a sample and effectively, exclude from view any low resistance components such as those associated to inhomogenous samples that may have conductive grain cores but resistive grain boundaries. Therefore, it is important to determine the resistance of a region or the total resistance of the by modelling the impedance response to an equivalent circuit $^{[40]}$.

Figure 1: Impedance spectra for $\mathrm{CeO}_{2}$ measured at $450{ }^{\circ} \mathrm{C}$. (a) Experimental data shown for impedance complex plane plot; the frequencies of some data point are indicated. (b) C' spectroscopic plot, (c) Y' spectroscopic plot and (d) Z"'/M" spectroscopic plots.

The plot of $\log C^{\prime} / \log f$ Figure 1(b) for the same data shows
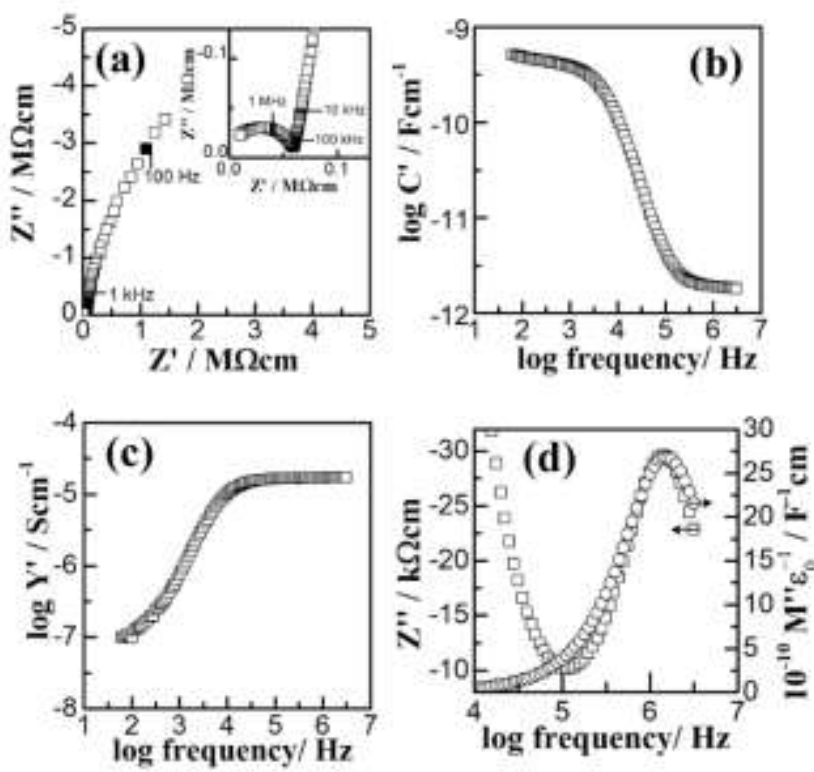

evidence for a high frequency plateau of magnitude $\sim 2 \mathrm{pFcm}^{-1}$ which corresponds to the values of the bulk permittivity, $\varepsilon_{b}$ of $\sim 23$, given by $\varepsilon_{b}=C^{\prime} / \varepsilon_{0}$ where $\varepsilon_{0}$ is the permittivity of free space, $8.854 \times 10^{-14} \mathrm{Fcm}^{-1}$. At lower frequencies, $C^{\prime}$ values increase with evidence of a second plateau at $\sim 600 \mathrm{pFcm}^{-1}$ which represents the grain boundary capacitance. The $Z$ "'/M" spectroscopic plots (d) show one main peak for $M$ " at high frequencies which coincides with a $Z$ " peak, which is an indicator of the electrical homogeneity of the sample. At lower frequencies a prominent high peak for Z" is observed, however the corresponding $M$ " peak is not seen. The peak maximum of an ideal, Debye-like $M$ " peak is inversely proportional to the capacitance of the $\mathrm{R}-\mathrm{C}$ element responsible for the peak; $M_{\max }^{\prime \prime}=C_{0} / 2 C$, where $C_{0}$ is the capacitance of the empty jig introduced above. Therefore, since the smallest capacitance usually represents the bulk component, the $M$ " and Z" peaks observed at high frequencies enable assignment of these peaks to the bulk. On representing the log $Y^{\prime} / \log f$ spectroscopic plot (c), the data shows slight dispersion at high frequencies with a frequency dependent power law dispersion. The data also give an indication of existence of another plateau at low frequencies.

In order to further interpret the obtained results, data were fitted to possible equivalent circuits at different temperatures, from 
$300{ }^{\circ} \mathrm{C}$ to $700{ }^{\circ} \mathrm{C}$ at every $50^{\circ} \mathrm{C}$ and at different atmospheres, Figures S1-S3 and table S1. The first step to fit impedance data is to establish the equivalent circuit at low temperature, where only the bulk response is detected. The second stage is to find the appropriate equivalent circuit to data obtained at higher temperatures where other features appear and therefore, additional elements are required.

The effect of different atmospheres on the impedance response is shown in Figure 2. Even though at $400{ }^{\circ} \mathrm{C}$ only the bulk response is observed, impedance data show significant differences as a function of the atmosphere. As expected $\mathrm{CeO}_{2}$ shows $\mathrm{n}$-type conductivity, i.e. under $\mathrm{N}_{2}$ or reducing atmospheres (CO) the bulk resistivity decreases and under oxidizing conditions the bulk resistivity increases, Figure $2 \mathrm{a}$. Changes in conductivity in reducing atmospheres are known to be a consequence of extrinsic electronic conduction in materials because of loss of oxygen, which leads to changes in the carrier concentration of electrons and the creation of oxygen vacancies. The mechanism can be easily described using the Kröger-Vink notation, equation 4:

$O_{O}^{x} \rightarrow 1 / 2 O_{2}+V_{O}^{*}+2 e^{\prime}$

For thermodynamic reasons, carbon dioxide can be considered as an inert gas at low temperature and therefore a decrease of the bulk resistivity should be expected. Conversely, the bulk resistivity under $\mathrm{CO}_{2}$ increases and it is ever larger than under pure $\mathrm{O}_{2}$.
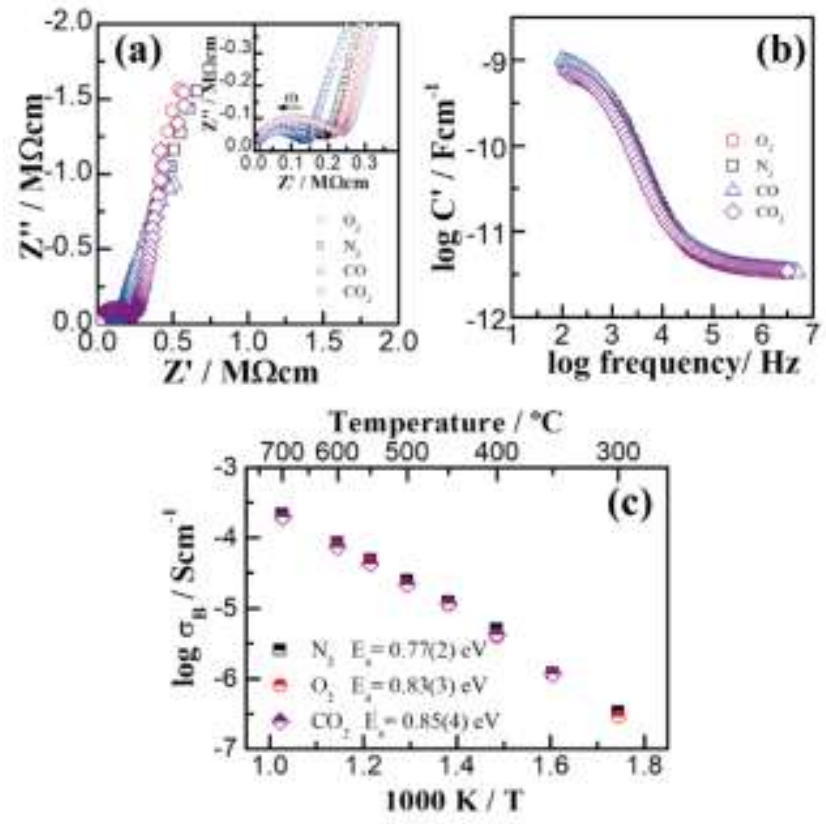

Figure 2: (a) Impedance complex plane plots and (b) C' spectroscopic plots at $400{ }^{\circ} \mathrm{C}$ as a function of the atmosphere. (c) Arrhenius plots for bulk conductivity $\left(\sigma_{b}\right)$ as a function of the atmosphere.

Recently, it has been shown that $\mathrm{CO}_{2}$ can act as a soft oxidant, oxidizing $\mathrm{Ce}^{3+}$ to $\mathrm{Ce}^{4+[41-43]}$. Although the oxidizing power of carbon dioxide is lower than that of pure oxygen, it is faster than the diffusional migration of oxygen from the bulk ${ }^{[41]}$. Moreover, DFT calculations have corroborated the basic character of the $\mathrm{CeO}_{2}$ surface by the observation of a charge transfer of $0.46 \mathrm{e}^{-}$ from ceria to the monodentate $\mathrm{CO}_{2}$ adsorbed species ${ }^{[44]}$.
Additionally, it has been shown that under specific conditions, like under pure $\mathrm{O}_{2}$ atmosphere, anionic species such as superoxide or peroxide are present in the surface of $\mathrm{CeO}_{2}$ and other oxides which may contribute to promote electronic conduction and therefore, a decrease of the resistivity [45-49]. The activation energies extracted from the Arrhenius plots of $\mathrm{CeO}_{2}$, where $\sigma_{b}=1 / R_{b}$ obtained from the bulk resistance in $Z^{*}$ plots, show slight differences as a function of the atmosphere, Figure 2c.

The activation energies of data recorded under $\mathrm{CO}_{2}$ or $\mathrm{O}_{2}$ are higher than those measured under $\mathrm{N}_{2}$ or $\mathrm{CO}$. The later must contain a contribution of both electronic and ionic conductivities. From the non-parallel Arrhenius plots together with the lower activation energy $(\sim 0.1 \mathrm{eV})$ observed in $\mathrm{N}_{2}$ atmosphere it can be deduced that the conduction mechanisms operating at different atmospheres may be slightly different. This is because conductivity, $\sigma$, is given by the product of carrier concentration, $n$, electronic charge, $e$ and mobility, $\mu$, i.e. $\sigma=n e \mu$. Therefore, as described in equation 4 , the increase of resistivity under $\mathrm{O}_{2}$ and $\mathrm{CO}_{2}$, oxidizing atmospheres, can be explained in terms of a decrease of the carrier concentration, electrons and oxygen vacancies, and a change in the conduction mechanism. However, the data in $\mathrm{CO}_{2}$ or $\mathrm{O}_{2}$ may also contain small electronic component. We have not performed exhaustive analysis of the electronic transport number in $\mathrm{CeO}_{2}$ as a function of the atmosphere or temperature that would allow to better understand the contribution of each conduction mechanism to the electrical response of the material. Unlike the differences observed in the $Z$ complex plane plot, the C' plot does not show significant differences as a function of the atmosphere (Figure $2 b$ ).

The catalytic performance of the $\mathrm{CeO}_{2}$ pellets was analyzed in terms of $\mathrm{CO}$ oxidation. Figure 3 shows the $\mathrm{CO}$ conversion of the $\mathrm{CeO}_{2}$ pellets used in EIS measurements and of the empty reactor (blank run), for comparison.

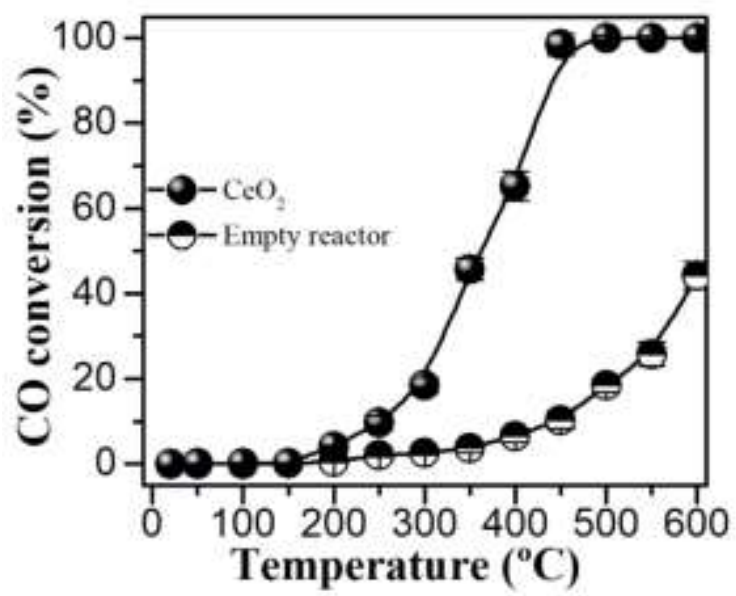

Figure 3: Catalytic performance of $\mathrm{CO}$ oxidation, conversion over $\mathrm{CeO}_{2}$ pellet and over an empty reactor (blank).

Results show a $100 \%$ conversion of $\mathrm{CO}$ to $\mathrm{CO}_{2}$ at $450{ }^{\circ} \mathrm{C}$ and a temperature of half conversion $\left(\mathrm{T}_{50}\right)$ of $\mathrm{CO}$ at around $350{ }^{\circ} \mathrm{C}$. It should be noted that at this temperature, the $\mathrm{CO}$ conversion of the blank run achieves less than $5 \%$, therefore the main contribution to the catalytic oxidation of $\mathrm{CO}$ is the $\mathrm{CeO}_{2}$. Moreover, it is important to mention that typically the catalytic performance of any material is analyzed using powdered samples which are, 
generally, nanometric ${ }^{[23,27]}$. When using pellets, the surface exposed to the gases is much lower than the surface exposed using powders in the nanosize. Hence, the obtained results using pellets differ by a shift in temperature of $50-100^{\circ} \mathrm{C}$ compared with the results of $\mathrm{CeO}_{2}$ nanopowder depending on their size and morphology [26].

It is common when analyzing the catalytic performance of a catalyst to collect data after reaching a steady state; this is necessary because the temperature of the catalyst needs to stabilize. This effect can be monitored with impedance spectroscopy as can be seen in Figure 4 . The reactor prepared for impedance analysis was set to static mode at $450{ }^{\circ} \mathrm{C}$, i.e. the reactor was firstly purged with a gas mixture of $\mathrm{CO}: \mathrm{O}_{2}=1: 1$ and then properly closed $(\sim 1 \mathrm{~atm})$ to obtain a static atmosphere. The impedance of the sample was monitored as a function of time. As the reactor chamber is closed, the $\mathrm{CeO}_{2}$ catalyst oxidizes $\mathrm{CO}$ and therefore, the impedance of the sample changes. At first stages, the reducing conditions are more evident, the concentration of $\mathrm{CO}$ is high, and the resistivity of the bulk is rather low. However, as the chamber mixture evolves and the concentration of $\mathrm{CO}$ decreases by increasing the concentration of $\mathrm{CO}_{2}$, the bulk resistivity increases reaching a steady state after $\sim 30 \mathrm{~min}$. No further evolution of the resistivity is observed during observations for a period of up to $90 \mathrm{~min}$. It is important to mention that the stabilization time of a nanopowder sample could be shorter due to the larger superficial area of the catalyst. The resistivity values do not reach the values obtained under pure $\mathrm{CO}_{2}$ even if the conversion is $\sim 100 \%$ as the initial mixture had an excess of oxygen. Therefore, the resistivity values at the steady state are between the resistivity values of pure $\mathrm{CO}_{2}$ and $\mathrm{O}_{2}$. The sensitivity of the sample and its impedance to the surrounding atmosphere is evident, the resistivity values reached at the steady state are closer to the resistivity values under pure $\mathrm{CO}_{2}$, as the final atmosphere is richer in $\mathrm{CO}_{2}$ than in $\mathrm{O}_{2}$.

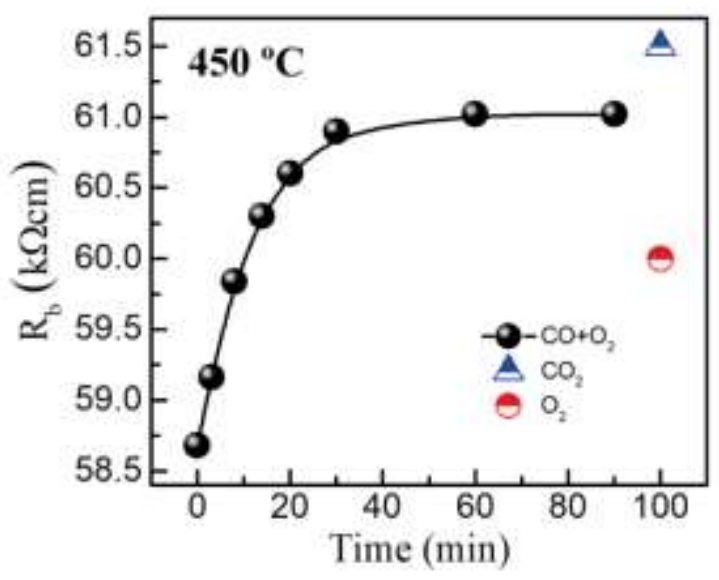

Figure 4: Evolution of $R_{b}$ as a function of the atmosphere and time in static mode at $450^{\circ} \mathrm{C}$.

The sensitivity of the sample to the surrounding atmosphere allows to follow the catalytic reaction as a function of the $\mathrm{CO}: \mathrm{O}_{2}$ ratio. Figure 5 shows the evolution of the $\mathrm{CO}$ conversion and the evolution of the $\mathrm{CeO}_{2}$ bulk resistivity as a function of the $\mathrm{CO}: \mathrm{O}_{2}$ ratio at $450{ }^{\circ} \mathrm{C}$. For comparison purposes the resistivity of the sample under pure $\mathrm{CO}_{2}(100 \%)$ and pure $\mathrm{O}_{2}(100 \%)$ are also shown.

The bulk resistivity and the CO conversion show the same tendency. The conversion of $\mathrm{CO}$ increases when the $\mathrm{CO} / \mathrm{O}_{2}$ ratio is $\leq 1$ and shows a maximum of $\sim 100 \%$ at a $\mathrm{CO} / \mathrm{O}_{2}$ ratio of 1 . In this region all the $\mathrm{CO}$ is consumed and the only product of the reaction is $\mathrm{CO}_{2}$ together with an excess of $\mathrm{O}_{2}$. The bulk resistivity shows exactly the same evolution; it increases up to a maximum which coincides with the maximum of conversion. As the surrounding atmosphere is mainly $\mathrm{CO}_{2}$ and $\mathrm{O}_{2}$, the resistivity of $\mathrm{CeO}_{2}$ remains close to the resistivity values when the impedance is measured under pure $\mathrm{CO}_{2}$. When the $\mathrm{CO} / \mathrm{O}_{2}$ ratio is $>1$, as expected, the $\mathrm{CO}$ conversion drops drastically as there is an excess of $\mathrm{CO}$ and the whole $\mathrm{O}_{2}$ is consumed. The same trend is

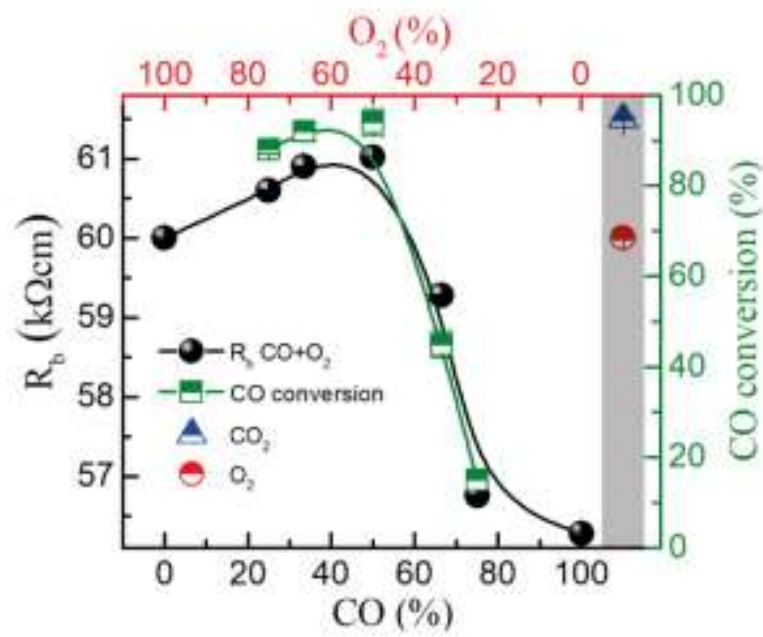

observed in sample's bulk impedance behavior, namely a drastic decrease of the resistivity is observed.

Figure 5: Evolution of $\mathrm{Rb}$ and conversion over $\mathrm{CeO}_{2}$ pellet as a function of the $\mathrm{CO}: \mathrm{O}_{2}$ ratio at $450{ }^{\circ} \mathrm{C}$. The resistivity of the sample under $100 \% \mathrm{CO}_{2}$ and 100 $\mathrm{O}_{2}$ are also shown as half white/blue triangle and half red/white circle, respectively

\section{Conclusion}

In summary, ceria polycrystals in the form of dense pellets have been deeply analyzed by impedance spectroscopy technique under catalytic $\mathrm{CO}$ oxidation conditions. The prepared ceramics show a typical impedance response with a homogenous electrical behavior under different atmospheres. Results show that $\mathrm{CeO}_{2}$ is very sensitive to the surrounding atmosphere and its $n$-type conductivity is confirmed. Moreover, even though the catalyst response of ceria was analyzed in the form of pellets of a density $\sim 80 \%$, the catalytic response of the ceramic is comparable to the catalytic response of ceria nanopowder. The most interesting aspect of this study is that the catalytic performance of ceria can be easily followed by impedance spectroscopy, namely the conversion of $\mathrm{CO}$ could be determined from the bulk resistivity within a simple impedance analysis. Most importantly, combining catalytic tests and electrochemical impedance spectroscopy represents a novel and powerful approach to infer on the transport properties of ceria and/or modified ceria under reaction conditions, which may allow a better understanding of the mechanisms involved. These results open the possibility to use this technique 
in new applications and also to further study other catalytic materials.

\section{Experimental Section}

Nanopolycrystalline $\mathrm{CeO}_{2}$ was prepared by conventional precipitation method. A $40 \mathrm{ml} 26 \%$ ammonia $\left(\mathrm{NH}_{3}\right)$ solution was added dropwise to a $300 \mathrm{ml}$ solution of $12.6 \mathrm{~g}$ of cerium nitrate $\left(\mathrm{Ce}\left(\mathrm{NO}_{3}\right)_{3} \cdot 6 \mathrm{H}_{2} \mathrm{O}\right.$, Alfa Aesar) under vigorous stirring, reaching a $\mathrm{pH}$ value of $\sim 9$. The formed precipitate was filtered and washed with distilled water until a neutral $\mathrm{pH}$ was reached. The powder was dried at $100^{\circ} \mathrm{C}$ overnight and calcined at $450{ }^{\circ} \mathrm{C}$ for $4 \mathrm{~h}$.

In order to obtain pellets with a relative density of $\sim 80 \%$, the calcined powders were mixed with a $5 \mathrm{wt} \%$ of polyvinyl alcohol (PVA) as a binder and pressed into disks of $\sim 10 \mathrm{~mm}$ diameter and $\sim 1 \mathrm{~mm}$ thickness using a uniaxial hydraulic press under a pressure of $400 \mathrm{MPa}$. The green pellets were treated at $500{ }^{\circ} \mathrm{C}$ for $4 \mathrm{~h}$ to remove the binder and densify. The final weight of the samples was $\sim 0.4 \mathrm{~g}$ and their relative density was of $\sim 80 \%$. Phase purity was examined at room temperature by X-ray diffraction (XRD) on crushed samples using a Bruker D8 advance system with $\mathrm{Cu}_{\mathrm{a}}$ radiation (45 kV, $35 \mathrm{~mA}$ ) in a Bragg-Brentano geometry (not shown).

For electrical property measurements, gold electrodes were partially sputtered on both sides of the pellets. The samples were placed in a jig with two-electrode configuration in an in-house furnace which allows to control the atmosphere of the reactor chamber. Measurements were made with an impedance analyzer, HP4192A, in the frequency range $5 \mathrm{~Hz}-3$ $\mathrm{MHz}$, the nominal ac voltage used was $100 \mathrm{mV}$ and no $d c$ voltage was applied during the impedance measurements. Samples were analyzed under static or dynamic dried atmospheres of $\mathrm{O}_{2}(99.999 \%), \mathrm{N}_{2}(99.999 \%)$, $\mathrm{CO}_{2}(99.999 \%), \mathrm{CO}(99.9 \%)$ or combination of $\mathrm{CO}: \mathrm{O}_{2}$ with a total flowrate of $30 \mathrm{ml} / \mathrm{min}$ from room temperature to $700^{\circ} \mathrm{C}$. At each temperature, the system was allowed to equilibrate for $1 \mathrm{~h}$, prior to impedance measurements. Impedance data were corrected for sample geometry and electrode contact area; this allowed resistance and capacitance to be reported in resistivity and permittivity units of $\Omega \mathrm{cm}$ and $\mathrm{F} \mathrm{cm}^{-1}$, respectively Open circuit measurements of an empty jig were used to obtain the blank parallel capacitance, $C_{0}$, of the jig and leads, which was subtracted from the values obtained with a sample present in the jig. In order to obtain the $C_{0}$ value, the jig was assembled with electrodes of similar dimension, but without a sample in place. Closed circuit measurements were obtained by connecting the two electrodes directly and used to correct for the series jig resistance. Corrected data were analyzed and modelled using Zview (Scriber Associates Inc.) software. Details of the impedance analysis can be found extendedly in the literature ${ }^{[39,50-53]}$. For the sake of convenience, we have added a brief introduction to EIS and to the analysis of EIS data in the supplementary information file.

The catalytic tests of $\mathrm{CO}$ oxidation were performed over the same partially Au covered samples used in EIS, for statistical reason three different samples were tested. The catalytic tests were performed from room temperature up to $600{ }^{\circ} \mathrm{C}$ by increasing the reactor temperature in steps of $20^{\circ} \mathrm{C}$, until steady state was reached. A total flowrate of $30 \mathrm{ml} / \mathrm{min}$ was used. A gas chromatograph (Micro GC Agilent 3000A) equipped with MS $5 \AA$, Plot $U$ and Stabilwax capillary columns and TCD detectors was used to measure on-line gas concentrations every $4 \mathrm{~min}$. The product of the reaction was $\mathrm{CO}_{2}$ and the remaining $\mathrm{CO}$ and $\mathrm{O}_{2}$ reactants were also measured. The CO conversion $(X \mathrm{CO})$ was calculated using equation 5 ,

$\chi_{C O}(\%)=\frac{n_{C O, \text { in }}-n_{C O, \text { out }}}{n_{C O, \text { in }}} \cdot 100=\frac{n_{C O_{2}, \text { out }}}{n_{C O, \text { in }}} \cdot 100$

where $n_{C O, \text { in }}$ is the inlet molar flowrate of $\mathrm{CO}, n_{C O, o u t}$ is the outlet molar flowrate of $\mathrm{CO}$ that has not been reacted and $n_{\mathrm{CO}_{2} \text {,out }}$ is the outlet molar flowrate of the $\mathrm{CO}_{2}$ produced during the experiment.

\section{Acknowledgements}

This work has been funded by projects MICINN/FEDER RTI2018093996-B-C31, GC 2017 SGR 128 and MAT2016-80410-PI. JL is a Serra Húnter fellow and is grateful to ICREA Academia program. $\mathrm{XV}$ is grateful to Spanish Government, Ministerio de Ciencia, Innovación y Universidades Juan de la Cierva-Incorporación program for an individual fellowship grant agreement IJCI-201731449.

Keywords: Electrochemical Impedance Spectroscopy • ceria • $\mathrm{CO}$ oxidation $\cdot$ oxygen vacancies $\cdot$ transport properties

[1] M. Mogensen, T. Lindeaaard, U. R. Hansen, G. Mogensen, J. Electrochem. Soc. 1994, 141, 2122-2128.

[2] H. Inaba, Solid State Ionics 1996, 83, 1-16.

[3] J. W. Fergus, J. Power Sources 2006, 162, 30-40.

[4] J. Prado-Gonjal, R. Schmidt, J. Espíndola-Canuto, P. RamosAlvarez, E. Morán, J. Power Sources 2012, 209, 163-171.

[5] N. Izu, W. Shin, N. Murayama, S. Kanzaki, Sensors Actuators, $B$ Chem. 2002, 87, 95-98.

[6] E. Laubender, N. B. Tanvir, O. Yurchenko, G. Urban, Procedia Eng. 2015, 120, 1058-1062.

[7] T. L. Simonenko, N. P. Simonenko, A. S. Mokrushin, E. P. Simonenko, O. V. Glumov, N. A. Mel'nikova, I. V. Murin, M. V. Kalinina, O. A. Shilova, V. G. Sevastyanov, N. T. Kuznetsov, Ceram. Int. 2020, 46, 121-131.

[8] S. Park, J. M. Vohs, R. J. Gorte, Nature 2000, 404, 265-267.

[9] F. S. Torknik, M. Keyanpour-Rad, A. Maghsoudipour, G. M. Choi, Ceram. Int. 2014, 40, 1341-1350.

[10] Z. Qiao, C. Xia, Y. Cai, M. Afzal, H. Wang, J. Qiao, B. Zhu, J. Power Sources 2018, 392, 33-40.

[11] C. Hua, X. Fang, Z. Yang, Y. Gao, Z. Wang, L. Chen, Electrochem. commun. 2012, 25, 66-69.

[12] Z. Han, F. Kong, X. He, S. Tao, X. Jiang, B. Qian, J. Phys. Chem. Solids 2019, 134, 187-192.

[13] Q. Su, L. Chang, J. Zhang, G. Du, B. Xu, J. Phys. Chem. C 2013, 117, 4292-4298.

[14] L. G. A. Carvalho, L. A. Rocha, J. M. M. Buarque, R. R. Gonçalves, C. S. Nascimento, M. A. Schiavon, S. J. L. Ribeiro, J. L. Ferrari, J. Lumin. 2015, 159, 223-228.

[15] A. Corma, P. Atienzar, H. García, J. Y. Chane-Ching, Nat. Mater. 2004, 3, 394-397.

[16] Y. Liu, Y. Qiao, W.-X. Zhang, Z. Li, X.-L. Hu, L.-X. Yuan, Y.-H. Huang, J. Mater. Chem. 2012, 22, 24026.

[17] I. Lucentini, A. Casanovas, J. Llorca, Int. J. Hydrogen Energy 2019, 44, 12693-12707.

[18] H. Xing, G. Long, J. Zheng, H. Zhao, Y. Zong, X. Li, Y. Wang, X. Zhu, M. Zhang, X. Zheng, Electrochim. Acta 2020, 337, 135817.

[19] C. Liu, H. Sun, J. Qian, Z. Chen, F. Chen, S. Liu, Y. Lv, X. Lu, A. Chen, J. Alloys Compd. 2017, 722, 54-59.

[20] Z. Wu, M. Li, S. H. Overbury, J. Catal. 2012, 285, 61-73.

[21] E. Aneggi, J. Llorca, M. Boaro, A. Trovarelli, J. Catal. 2005, 234, 88-95.

[22] C. Ho, J. C. Yu, T. Kwong, A. C. Mak, S. Lai, Chem. Mater. 2005, 17, 4514-4522.

[23] L. Soler, A. Casanovas, A. Urrich, I. Angurell, J. Llorca, Appl. Catal. B Environ. 2016, 197, 47-55. 
A. Trovarelli, J. Llorca, ACS Catal. 2017, 7, 4716-4735.

J. Qin, J. Lu, M. Cao, C. Hu, Nanoscale 2010, 2, 2739-2743.

W. Zou, C. Ge, M. Lu, S. Wu, Y. Wang, J. Sun, Y. Pu, C. Tang, F. Gao, L. Dong, RSC Adv. 2015, 5, 98335-98343.

[27] X. Zhang, K. Li, W. Shi, C. Wei, X. Song, S. Yang, Z. Sun, Nanotechnology 2017, 28, 045602.

[28] E. M. Slavinskaya, R. V Gulyaev, A. V Zadesenets, O. A. Stonkus, V. I. Zaikovskii, "Applied Catal. B, Environ. 2015, 166-167, 91-103. G. Nurk, K. Kooser, S. Urpelainen, T. Käämbre, U. Joost, M. Kodu, I. Kivi, R. Kanarbik, E. Kukk, E. Lust, J. Power Sources 2018, 378, 589-596.

[30] F. Bidrawn, G. Kim, G. Corre, J. T. S. Irvine, J. M. Vohs, R. J. Gorte, Electrochem. Solid-State Lett. 2008, 11, 167-170.

[31] Z. Huang, Z. Zhao, H. Qi, X. Wang, B. Tu, M. Cheng, J. Energy Chem. 2020, 40, 46-51.

[32] Z. A. Feng, F. El Gabaly, X. Ye, Z. X. Shen, W. C. Chueh, Nat. Commun. 2014, 5, DOI 10.1038/ncomms5374.

[33] K. Kooser, T. Käämbre, M. Vestli, U. Joost, S. Urpelainen, M. Kook, F. Bournel, J. J. Gallet, E. Lust, E. Kukk, G. Nurk, Int. J. Hydrogen Energy 2020, 5, DOI 10.1016/j.ijhydene.2020.06.228.

[34] N. I. Ionescu, M. Câldâraru, React. Kinet. Catal. Lett. 1978, 8, 477481.

[35] A. Vasile, V. Bratan, C. Hornoiu, M. Caldararu, N. I. Ionescu, T. Yuzhakova, Á. Rédey, Appl. Catal. B Environ. 2013, 140-141, 2531.

[36] A. Vasile, C. Hornoiu, V. Munteanu, N. I. Ionescu, T. Yuzhakova, A. Rédey, Revenue Rom. Chim. 2016, 61, 503-509.

[37] M. Breysse, M. Guening, B. Claudel, H. Latreille, J. Véron, J. Catal. 1972, 27, 275-280

[38] P. Chesler, C. Hornoiu, V. Bratan, C. Munteanu, G. Postole, N. I. Ionescu, T. Juzsakova, A. Redey, M. Gartner, React. Kinet. Mech. Catal. 2016, 117, 551-563.

[39] J. T. S. Irvine, D. C. Sinclair, A. R. West, Adv. Mater. 1990, 2, 132138.

[40] M. A. Hernández, N. Masó, A. R. West, Appl. Phys. Lett. 2016, 108, 152901.

[41] O. Demoulin, M. Navez, J. L. Mugabo, P. Ruiz, Appl. Catal. B Environ. 2007, 70, 284-293.

[42] D. Yamaguchi, L. Tang, N. Scarlett, K. Chiang, Chem. Eng. Sci. 2020, 217, 115520.

[43] G. F. Fine, L. M. Cavanagh, A. Afonja, R. Binions, Sensors 2010, 10, 5469-5502.

[44] U. Tumuluri, G. Rother, Z. Wu, Ind. Eng. Chem. Res. 2016, 55, 3909-3919.

[45] P. J. Gellings, H. J. M. Bouwmeester, Catal. Today 1992, 12, 1101.

[46] V. V. Pushkarev, V. I. Kovalchuk, J. L. D'Itri, J. Phys. Chem. B 2004, 108, 5341-5348.

[47] J. Xu, J. Harmer, G. Li, T. Chapman, P. Collier, S. Longworth, S. C. Tsang, Chem. Commun. 2010, 46, 1887-1889.

[48] X. Vendrell, A. R. West, J. Am. Ceram. Soc. 2019, 102, 6100-6106.

[49] P. Ren, N. Masó, A. R. West, Phys. Chem. Chem. Phys. 2013, 15, 20943.

[50] I. M. Hodge, M. D. Ingram, A. R. West, J. Electroanal. Chem. Interfacial Electrochem. 1976, 74, 125-143.

[51] D. C. Sinclair, Bol. la Soc. Esp. Ceram. y Vidr. 1995, 65, 55-66.

[52] E. J. Abram, D. C. Sinclair, A. R. West, J. Electroceramics 2003, 10,
165-177.

[53] X. Vendrell, A. R. West, J. Electrochem. Soc. 2018, 165, F966F975. 
